\title{
Existence of Solutions of $\alpha \in(2,3]$ Order Fractional Three-Point Boundary Value Problems with Integral Conditions
}

\author{
N. I. Mahmudov and S. Unul \\ Eastern Mediterranean University, Gazimagusa, Mersin 10, Turkey \\ Correspondence should be addressed to N. I. Mahmudov; nazim.mahmudov@emu.edu.tr \\ Received 16 April 2014; Revised 20 June 2014; Accepted 20 June 2014; Published 6 July 2014 \\ Academic Editor: Juan J. Nieto \\ Copyright ( 2014 N. I. Mahmudov and S. Unul. This is an open access article distributed under the Creative Commons Attribution \\ License, which permits unrestricted use, distribution, and reproduction in any medium, provided the original work is properly cited. \\ Existence and uniqueness of solutions for $\alpha \in(2,3]$ order fractional differential equations with three-point fractional boundary \\ and integral conditions involving the nonlinearity depending on the fractional derivatives of the unknown function are discussed. \\ The results are obtained by using fixed point theorems. Two examples are given to illustrate the results.
}

\section{Introduction}

Recently, the theory on existence and uniqueness of solutions of linear and nonlinear fractional differential equations has attracted the attention of many authors; see, for example, [119 ] and references therein. Many of the physical systems can better be described by integral boundary conditions. Integral boundary conditions are encountered in various applications such as population dynamics, blood flow models, chemical engineering, and cellular systems. Moreover, boundary value problems with integral boundary conditions constitute a very interesting and important class of problems. They include two-point, three-point, multipoint, and nonlocal boundary value problems as special cases. The existing literature mainly deals with first order and second order boundary value problems and there are a few papers on third order problems.

El-Shahed [14] studied existence and nonexistence of positive solution of nonlinear fractional two-point boundary value problem:

$$
\begin{gathered}
\mathfrak{D}_{0^{+}}^{\alpha} u(t)+\lambda a(t) f(u(t))=0, \quad 0<t<1 ; 2<\alpha<3, \\
u(0)=u^{\prime}(0)=u^{\prime}(1)=0,
\end{gathered}
$$

where $\mathfrak{D}_{0^{+}}^{\alpha}$ denotes the Caputo derivative of fractional order $\alpha, \lambda$ is a positive parameter, and $a:(0,1) \rightarrow[0, \infty)$ is continuous function.

In [8], Ahmad and Ntouyas studied a boundary value problem of nonlinear fractional differential equations of order $\alpha \in(2,3]$ with antiperiodic type integral boundary conditions:

$$
\begin{gathered}
\mathfrak{D}_{0^{+}}^{\alpha} u(t)=f(t, u(t)) ; \quad 0<t<T ; 2<\alpha \leq 3, \\
u^{(j)}(0)-\lambda_{j} u^{(j)}(T)=\mu_{j} \int_{0}^{T} g_{j}(s, u(s)) d s, \quad j=0,1,2,
\end{gathered}
$$

where $\mathfrak{D}_{0^{+}}^{\alpha}$ denotes the Caputo derivative of fractional order $\alpha, u^{(j)}$ denotes $j$ th derivative of $u, f, g_{0}, g_{1}, g_{2}:[0, T] \times \mathbb{R} \rightarrow$ $\mathbb{R}$ are given continuous functions, and $\lambda_{j}, \mu_{j} \in \mathbb{R}\left(\lambda_{j} \neq 1\right)$. The same problem for fractional differential inclusions is considered in [9].

Ahmad and Nieto [7] studied existence and uniqueness results for the following general three-point fractional boundary value problem involving a nonlinear fractional differential equation of order $\alpha \in(m-1, m]$ :

$$
\begin{gathered}
\mathfrak{D}_{0^{+}}^{\alpha} u(t)=f(t, u(t)) ; \quad 0<t<T, m \geq 2, \\
u(0)=u^{\prime}(0)=\cdots=u^{(m-2)}(0)=0, \quad u(1)=\lambda u(\eta) .
\end{gathered}
$$

However, very little work has been done on the case when the nonlinearity $f$ depends on the fractional derivative of the unknown function. Su and Zhang [17] and Rehman et al. [16] studied the existence and uniqueness of solutions for following nonlinear two-point and three-point fractional 
boundary value problem when the nonlinearity $f$ depends on the fractional derivative of the unknown function.

In this paper, we investigate the existence (and uniqueness) of solution for nonlinear fractional differential equations of order $\alpha \in(2,3]$ when the nonlinearity $f$ depends on the fractional derivatives of the unknown function

$$
\begin{array}{r}
\mathfrak{D}_{0^{+}}^{\alpha} u(t)=f\left(t, u(t), \mathfrak{D}_{0^{+}}^{\beta_{1}} u(t), \mathfrak{D}_{0^{+}}^{\beta_{2}} u(t)\right) ; \\
0 \leq t \leq T ; \quad 2<\alpha \leq 3
\end{array}
$$

with the three-point and integral boundary conditions

$$
\begin{gathered}
a_{0} u(0)+b_{0} u(T)=\lambda_{0} \int_{0}^{T} g_{0}(s, u(s)) d s, \\
a_{1} \mathfrak{D}_{0^{+}}^{\beta_{1}} u(\eta)+b_{1} \mathfrak{D}_{0^{+}}^{\beta_{1}} u(T)=\lambda_{1} \int_{0}^{T} g_{1}(s, u(s)) d s, \\
0<\beta_{1} \leq 1, \quad 0<\eta<T, \\
a_{2} \mathfrak{D}_{0^{+}}^{\beta_{2}} u(\eta)+b_{2} \mathfrak{D}_{0^{+}}^{\beta_{2}} u(T)=\lambda_{2} \int_{0}^{T} g_{2}(s, u(s)) d s, \\
1<\beta_{2} \leq 2,
\end{gathered}
$$

where $\mathfrak{D}_{0^{+}}^{\alpha}$ denotes the Caputo fractional derivative of order $\alpha, f, g_{i}$ are continuous functions, and $a_{i}, b_{i}, \lambda_{i} \in \mathbb{R}$, for $i=$ $0,1,2$.

\section{Preliminaries}

Let us recall some basic definitions [20-22].

Definition 1. The Riemann Liouville fractional integral of order $\alpha$ for continuous function $f:[0, \infty) \rightarrow \mathbb{R}$ is defined as

$$
I_{0^{+}}^{\alpha} f(t)=\frac{1}{\Gamma(\alpha)} \int_{0}^{t}(t-s)^{\alpha-1} f(s) d s, \quad \alpha>0,
$$

provided the integral exists.

Definition 2. For $(n-1)$-times absolutely continuous function $f:[0, \infty) \rightarrow \mathbb{R}$, the Caputo derivative fractional order $\alpha$ is defined as

$$
\begin{array}{r}
\mathfrak{D}_{0^{+}}^{\alpha} f(t)=\frac{1}{\Gamma(n-\alpha)} \int_{0}^{t}(t-s)^{n-\alpha-1} f^{(n)}(s) d s \\
n-1<\alpha<n, \quad n=[\alpha]+1,
\end{array}
$$

where $[\alpha]$ denotes the integral part of the real number $\alpha$.

Lemma 3. Let $\alpha>0$. Then, the differential equation $\mathfrak{D}_{0^{+}}^{\alpha} f(t)=0$ has solutions

$$
\begin{gathered}
f(t)=k_{0}+k_{1} t+k_{2} t^{2}+\cdots+k_{n-1} t^{n-1}, \\
I_{0^{+}}^{\alpha} \mathfrak{D}_{0^{+}}^{\alpha} f(t)=f(t)+k_{0}+k_{1} t+k_{2} t^{2}+\cdots+k_{n-1} t^{n-1},
\end{gathered}
$$

where $k_{i} \in \mathbb{R}$ and $i=1,2,3, \ldots, n-1, n=[\alpha]+1$.
Caputo fractional derivative of order $n-1<\alpha<n$ for $t^{\gamma}$ is given as

$$
\mathfrak{D}_{0^{+}}^{\alpha} t^{\gamma}=\left\{\begin{array}{l}
\frac{\Gamma(\gamma+1)}{\Gamma(\gamma-\alpha+1)} t^{\gamma-\alpha} \\
\gamma \in \mathbb{N}, \gamma \geq n \text { or } \gamma \notin \mathbb{N}, \gamma>n-1 \\
0, \quad \gamma \in\{0,1, \ldots, n-1\} .
\end{array}\right.
$$

Assume that $\beta_{0}=0$ and

$$
\begin{gathered}
a_{0}+b_{0} \neq 0, \quad a_{1} \eta^{1-\beta_{1}}+b_{1} T^{1-\beta_{1}} \neq 0, \\
a_{i} \eta^{2-\beta_{i}}+b_{i} T^{2-\beta_{i}} \neq 0 .
\end{gathered}
$$

For convenience, we set

$$
\begin{aligned}
& \mu^{\beta_{1}}:=\frac{\Gamma\left(3-\beta_{1}\right)}{2\left(a_{1} \eta^{2-\beta_{1}}+b_{1} T^{2-\beta_{1}}\right)}, \\
& \mu^{\beta_{2}}:=\frac{\Gamma\left(3-\beta_{2}\right)}{2\left(a_{2} \eta^{2-\beta_{2}}+b_{2} T^{2-\beta_{2}}\right)}, \\
& \nu^{\beta_{1}}:=\frac{\Gamma\left(2-\beta_{1}\right)}{a_{1} \eta^{1-\beta_{1}}+b_{1} T^{1-\beta_{1}}}, \\
& \omega_{0}:=-\frac{1}{a_{0}+b_{0}}, \\
& \omega_{1}(t):=v^{\beta_{1}}\left(\frac{b_{0}}{a_{0}+b_{0}} T-t\right), \\
& \omega_{2}(t):=\frac{b_{0} T^{2}}{a_{0}+b_{0}} \mu^{\beta_{2}}-\frac{b_{0} T}{a_{0}+b_{0}} v^{\beta_{1}} \frac{\mu^{\beta_{2}}}{\mu^{\beta_{1}}}+v^{\beta_{1}} \frac{\mu^{\beta_{2}}}{\mu^{\beta_{1}}} t-\mu^{\beta_{2}} t^{2} .
\end{aligned}
$$

Lemma 4. For any $f, g_{0}, g_{1}, g_{2} \in C([0, T] ; \mathbb{R})$, the unique solution of the fractional boundary value problem

$$
\begin{gathered}
\mathfrak{D}_{0^{+}}^{\alpha} u(t)=f(t) ; \quad 0 \leq t \leq T, 2<\alpha \leq 3 \\
a_{0} u(0)+b_{0} u(T)=\lambda_{0} \int_{0}^{T} g_{0}(s) d s \\
a_{1} \mathfrak{D}_{0^{+}}^{\beta_{1}} u(\eta)+b_{1} \mathfrak{D}_{0^{+}}^{\beta_{1}} u(T)=\lambda_{1} \int_{0}^{T} g_{1}(s) d s \\
0<\eta<T, \quad 0<\beta_{1} \leq 1, \\
a_{2} \mathfrak{D}_{0^{+}}^{\beta_{2}} u(\eta)+b_{2} \mathfrak{D}_{0^{+}}^{\beta_{2}} u(T)=\lambda_{2} \int_{0}^{T} g_{2}(s) d s, \quad 1<\beta_{2} \leq 2,
\end{gathered}
$$


is given by

$$
\begin{aligned}
u(t)= & \int_{0}^{t} \frac{(t-s)^{\alpha-1}}{\Gamma(\alpha)} f(s) d s \\
& +\sum_{i=0}^{2} \omega_{i}(t) b_{i} \int_{0}^{T} \frac{(T-s)^{\alpha-\beta_{i}-1}}{\Gamma\left(\alpha-\beta_{i}\right)} f(s) d s \\
& +\sum_{i=1}^{2} \omega_{i}(t) a_{i} \int_{0}^{\eta} \frac{(\eta-s)^{\alpha-\beta_{i}-1}}{\Gamma\left(\alpha-\beta_{i}\right)} f(s) d s \\
& -\sum_{i=0}^{2} \omega_{i}(t) \lambda_{i} \int_{0}^{T} g_{i}(s) d s .
\end{aligned}
$$

Proof. By Lemma 3, for $2<\alpha \leq 3$, the general solution of the equation $\mathfrak{D}_{0^{+}}^{\alpha} u(t)=f(t)$ can be written as

$$
u(t)=\frac{1}{\Gamma(\alpha)} \int_{0}^{t}(t-s)^{\alpha-1} f(s) d s-k_{0}-k_{1} t-k_{2} t^{2}
$$

where $k_{0}, k_{1}, k_{2} \in \mathbb{R}$ are arbitrary constants. Moreover, by the formula (9), $\beta_{1}$ and $\beta_{2}$ order derivatives are as follows:

$$
\begin{aligned}
& \mathfrak{D}_{0^{+}}^{\beta_{1}} u(t)=I^{\alpha-\beta_{1}} f(t)-k_{1} \frac{t^{1-\beta_{1}}}{\Gamma\left(2-\beta_{1}\right)}-2 k_{2} \frac{t^{2-\beta_{1}}}{\Gamma\left(3-\beta_{1}\right)}, \\
& \mathfrak{D}_{0^{+}}^{\beta_{2}} u(t)=I^{\alpha-\beta_{2}} f(t)-2 k_{2} \frac{t^{2-\beta_{2}}}{\Gamma\left(3-\beta_{2}\right)} .
\end{aligned}
$$

Using boundary conditions (13), we get the following algebraic system of equations, for $k_{0}, k_{1}, k_{2}$,

$$
\begin{aligned}
& -\left(a_{0}+b_{0}\right) k_{0}-b_{0} T k_{1}-b_{0} T^{2} k_{2} \\
& =\lambda_{0} \int_{0}^{T} g_{0}(s) d s-b_{0} I_{0^{+}}^{\alpha} f(T), \\
& -\frac{a_{1} \eta^{1-\beta_{1}}+b_{1} T^{1-\beta_{1}}}{\Gamma\left(2-\beta_{1}\right)} k_{1}-2 \frac{a_{1} \eta^{2-\beta_{1}}+b_{1} T^{2-\beta_{1}}}{\Gamma\left(3-\beta_{1}\right)} k_{2} \\
& =\lambda_{1} \int_{0}^{T} g_{1}(s) d s-a_{1} I_{0^{+}}^{\alpha-\beta_{1}} f(\eta)-b_{1} I_{0^{+}}^{\alpha-\beta_{1}} f(T), \\
& -2 \frac{a_{2} \eta^{2-\beta_{2}}+b_{2} T^{2-\beta_{2}}}{\Gamma\left(3-\beta_{2}\right)} k_{2} \\
& =\lambda_{2} \int_{0}^{T} g_{2}(s) d s-a_{2} I_{0^{+}}^{\alpha-\beta_{2}} f(\eta)-b_{2} I_{0^{+}}^{\alpha-\beta_{2}} f(T) .
\end{aligned}
$$

Solving the above system of equations for $k_{0}, k_{1}, k_{2}$, we get the following:

$$
\begin{aligned}
& k_{2}=b_{2} \mu^{\beta_{2}} I_{0^{+}}^{\alpha-\beta_{2}} f(T)+a_{2} \mu^{\beta_{2}} I_{0^{+}}^{\alpha-\beta_{2}} f(\eta)-\lambda_{2} \mu^{\beta_{2}} \int_{0}^{T} g_{2}(s) d s, \\
& k_{1}=b_{1} v^{\beta_{1}} I_{0^{+}}^{\alpha-\beta_{1}} f(T)+a_{1} v^{\beta_{1}} I_{0^{+}}^{\alpha-\beta_{1}} f(\eta)-\lambda_{1} v^{\beta_{1}} \int_{0}^{T} g_{1}(s) d s \\
& -b_{2} \nu^{\beta_{1}} \frac{\mu^{\beta_{2}}}{\mu^{\beta_{1}}} I_{0^{+}}^{\alpha-\beta_{2}} f(T)-a_{2} \nu^{\beta_{1}} \frac{\mu^{\beta_{2}}}{\mu^{\beta_{1}}} I_{0^{+}}^{\alpha-\beta_{2}} f(\eta) \\
& +\lambda_{2} \nu^{\beta_{1}} \frac{\mu^{\beta_{2}}}{\mu^{\beta_{1}}} \int_{0}^{T} g_{2}(s) d s, \\
& k_{0}=\frac{b_{0}}{a_{0}+b_{0}} I_{0^{+}}^{\alpha} f(T)-\frac{\lambda_{0}}{a_{0}+b_{0}} \int_{0}^{T} g_{0}(s) d s \\
& -\frac{b_{0} b_{1} \nu^{\beta_{1}} T}{a_{0}+b_{0}} I_{0^{+}}^{\alpha-\beta_{1}} f(T)-\frac{b_{0} a_{1} \nu^{\beta_{1}} T}{a_{0}+b_{0}} I_{0^{+}}^{\alpha-\beta_{1}} f(\eta) \\
& +\frac{b_{0} \lambda_{1} \nu^{\beta_{1}} T}{a_{0}+b_{0}} \int_{0}^{T} g_{1}(s) d s+\frac{b_{0} b_{2} v^{\beta_{1}} T}{a_{0}+b_{0}} \frac{\mu^{\beta_{2}}}{\mu^{\beta_{1}}} I_{0^{+}}^{\alpha-\beta_{2}} f(T) \\
& +\frac{b_{0} a_{2} \nu^{\beta_{1}} T}{a_{0}+b_{0}} \frac{\mu^{\beta_{2}}}{\mu^{\beta_{1}}} I_{0^{+}}^{\alpha-\beta_{2}} f(\eta)-\frac{b_{0} \lambda_{2} \nu^{\beta_{1}} T}{a_{0}+b_{0}} \frac{\mu^{\beta_{2}}}{\mu^{\beta_{1}}} \int_{0}^{T} g_{2}(s) d s \\
& -\frac{b_{0} b_{2} \mu^{\beta_{2}} T^{2}}{a_{0}+b_{0}} I_{0^{+}}^{\alpha-\beta_{2}} f(T)-\frac{b_{0} a_{2} \mu^{\beta_{2}} T^{2}}{a_{0}+b_{0}} I_{0^{+}}^{\alpha-\beta_{2}} f(\eta) \\
& +\frac{b_{0} \lambda_{2} \mu^{\beta_{2}} T^{2}}{a_{0}+b_{0}} \int_{0}^{T} g_{2}(s) d s \text {. }
\end{aligned}
$$

Inserting $k_{0}, k_{1}, k_{2}$ into (15), we get the desired representation for the solution of (12)-(13).

Remark 5. The Green function of the BVP (4)-(5) is defined by

$$
G(t ; s)= \begin{cases}\frac{(t-s)^{\alpha-1}}{\Gamma(\alpha)}+G_{0}(t ; s), & 0 \leq s \leq t \leq T \\ G_{0}(t ; s), & 0 \leq t \leq s \leq T\end{cases}
$$

where

$$
\begin{aligned}
& G_{0}(t ; s)= \sum_{i=0}^{2} \omega_{i}(t) b_{i} \frac{(T-s)^{\alpha-\beta_{i}-1}}{\Gamma\left(\alpha-\beta_{i}\right)} \\
&+\sum_{i=1}^{2} \omega_{i}(t) a_{i} \frac{(\eta-s)^{\alpha-\beta_{i}-1}}{\Gamma\left(\alpha-\beta_{i}\right)} \chi_{(0, \eta)}(s), \\
& \chi_{(a, b)}(s):= \begin{cases}1, & s \in(a, b), \\
0, & s \notin(a, b) .\end{cases}
\end{aligned}
$$


Remark 6. For $\alpha=3, \beta_{1}=1, \beta_{2}=2$, and $\eta=0$, the BVP (4)-(5) can be written as follows:

$$
\begin{gathered}
u^{\prime \prime \prime}(t)=f\left(t, u(t), u^{\prime}(t), u^{\prime \prime}(t)\right), \quad 0 \leq t \leq T, \\
a_{0} u(0)+b_{0} u(T)=\lambda_{0} \int_{0}^{T} g_{0}(s, u(s)) d s, \\
a_{1} u^{\prime}(0)+b_{1} u^{\prime}(T)=\lambda_{1} \int_{0}^{T} g_{1}(s, u(s)) d s, \\
a_{2} u^{\prime \prime}(0)+b_{2} u^{\prime \prime}(T)=\lambda_{2} \int_{0}^{T} g_{2}(s, u(s)) d s .
\end{gathered}
$$

In this case, the Green function can be written as follows:

$$
G(t ; s)= \begin{cases}\frac{(t-s)^{2}}{\Gamma(\alpha)}+G_{0}(t ; s), & 0 \leq s \leq t \leq T, \\ G_{0}(t ; s), & 0 \leq t \leq s \leq T\end{cases}
$$

where

$$
\begin{aligned}
G_{0}(t ; s)= & \frac{b_{0}}{a_{0}+b_{0}} \frac{(T-s)^{2}}{\Gamma(\alpha)}+\left(-\frac{b_{0}}{a_{0}+b_{0}} \frac{b_{1}}{a_{1}+b_{1}} T+\frac{b_{1}}{a_{1}+b_{1}} t\right) \\
& \times \frac{T-s}{\Gamma(\alpha-1)} \\
& +\left(\frac{b_{0}}{a_{0}+b_{0}} \frac{b_{1}}{a_{1}+b_{1}} \frac{b_{2}}{a_{2}+b_{2}} T\right. \\
& \quad-\frac{b_{0}}{a_{0}+b_{0}} \frac{b_{2}}{2\left(a_{2}+b_{2}\right)} T^{2}-\frac{2 b_{1}}{a_{1}+b_{1}} \frac{b_{2}}{2\left(a_{2}+b_{2}\right)} t \\
& \left.+\frac{b_{2}}{2\left(a_{2}+b_{2}\right)} t^{2}\right) \frac{1}{\Gamma(\alpha-2)} .
\end{aligned}
$$

Moreover, the case

$$
\begin{array}{lll}
a_{0}=1, & b_{0}=0, & a_{1}=0, \\
b_{1}=1, & a_{2}=1, & b_{2}=0
\end{array}
$$

is investigated in [10]. In this case,

$$
G(t ; s)= \begin{cases}\frac{(t-s)^{2}}{\Gamma(\alpha)}+\frac{t(T-s)}{\Gamma(\alpha-1)}, & 0 \leq s \leq t \leq T, \\ \frac{t(T-s)}{\Gamma(\alpha-1)}, & 0 \leq t \leq s \leq T .\end{cases}
$$

\section{Existence and Uniqueness Results}

In this section we state and prove an existence and uniqueness result for the fractional BVP (4)-(5) by using the Banach fixed-point theorem. We study our problem in the space

$$
\begin{aligned}
& C_{\beta}([0, T] ; \mathbb{R}) \\
& \quad:=\left\{v \in C([0, T] ; \mathbb{R}): \mathfrak{D}_{0^{+}}^{\beta_{1}} v, \mathfrak{D}_{0^{+}}^{\beta_{2}} v \in C([0, T] ; \mathbb{R})\right\}
\end{aligned}
$$

equipped with the norm

$$
\|v\|_{\beta}:=\|v\|_{C}+\left\|\mathfrak{D}_{0^{+}}^{\beta_{1}} v\right\|_{C}+\left\|\mathfrak{D}_{0^{+}}^{\beta_{2}}\right\| \|_{C^{\prime}}
$$

where $\|\cdot\|_{C}$ is the sup norm in $C([0, T] ; \mathbb{R})$.

The following notations, formulae, and estimations will be used throughout the paper:

$$
\begin{aligned}
& \mathfrak{D}_{0^{+}}^{\beta_{1}} \omega_{1}(t)=-\frac{\nu^{\beta_{1}} t^{1-\beta_{1}}}{\Gamma\left(2-\beta_{1}\right)}, \\
& \mathfrak{D}_{0^{+}}^{\beta_{2}} \omega_{1}(t)=0, \\
& \mathfrak{D}_{0^{+}}^{\beta_{1}} \omega_{2}(t)=\frac{\nu^{\beta_{1}} \mu^{\beta_{2}} t^{1-\beta_{1}}}{\mu^{\beta_{1}} \Gamma\left(2-\beta_{1}\right)}-2 \frac{\mu^{\beta_{2}} t^{2-\beta_{1}}}{\Gamma\left(3-\beta_{1}\right)}, \\
& \mathfrak{D}_{0^{+}}^{\beta_{2}} \omega_{2}(t)=-2 \frac{\mu^{\beta_{2}} t^{2-\beta_{2}}}{\Gamma\left(3-\beta_{2}\right)}, \\
& \left|\omega_{0}\right|=\frac{1}{\left|a_{0}+b_{0}\right|}=: \rho_{0}, \\
& \left|\omega_{1}(t)\right| \leq\left|\nu^{\beta_{1}}\right|\left(\left|\omega_{0}\right|\left|b_{0}\right|+1\right) T=: \rho_{1}, \\
& \left|\omega_{2}(t)\right| \leq \frac{\left|b_{0}\right|\left|\mu^{\beta_{2}}\right|}{\left|a_{0}+b_{0}\right|} T^{2}+\frac{\left|b_{0}\right|\left|\nu^{\beta_{1}}\right|\left|\mu^{\beta_{2}}\right|}{\left|a_{0}+b_{0}\right|} \mid \frac{\mu^{\beta_{1}} \mid}{T} \\
& \quad+\frac{\left|\nu^{\beta_{1}}\right|\left|\mu^{\beta_{2}}\right|}{\left|\mu^{\beta_{1}}\right|} T+\left|\mu^{\beta_{2}}\right| T^{2}=: \rho_{2},
\end{aligned}
$$

$\widetilde{\rho}_{0}=0$,

$$
\begin{aligned}
& \left|\mathfrak{D}_{0^{+}}^{\beta_{1}} \omega_{1}(t)\right| \leq \frac{\left|\nu^{\beta_{1}}\right| T^{1-\beta_{1}}}{\Gamma\left(2-\beta_{1}\right)}=: \widetilde{\rho}_{1}, \\
& \left|\mathfrak{D}_{0^{+}}^{\beta_{1}} \omega_{2}(t)\right| \leq \frac{\left|\mu^{\beta_{2}}\right|\left|\nu^{\beta_{1}}\right| T^{1-\beta_{1}}}{\left|\mu^{\beta_{1}}\right| \Gamma\left(2-\beta_{1}\right)}+2 \frac{\left|\mu^{\beta_{2}}\right| T^{2-\beta_{1}}}{\Gamma\left(3-\beta_{1}\right)}=: \widetilde{\rho}_{2}, \\
& \widehat{\rho}_{0}=\widehat{\rho}_{1}=0, \\
& \left|\mathfrak{D}_{0^{+}}^{\beta_{2}} \omega_{2}(t)\right| \leq 2 \frac{\left|\mu^{\beta_{2}}\right| T^{2-\beta_{2}}}{\Gamma\left(3-\beta_{2}\right)}=: \widehat{\rho}_{2}, \\
& \Delta_{0}:=\frac{T^{\alpha-\tau}}{\Gamma(\alpha)}\left(\frac{1-\tau}{\alpha-\tau}\right)^{1-\tau} \\
& \quad+\sum_{i=0}^{2} \rho_{i}\left(\left|b_{i}\right| \frac{T^{\alpha-\beta_{i}-\tau}}{\Gamma\left(\alpha-\beta_{i}\right)}+\left|a_{i}\right| \frac{\eta^{\alpha-\beta_{i}-\tau}}{\Gamma\left(\alpha-\beta_{i}\right)}\right) \\
& \quad \times\left(\frac{1-\tau}{\alpha-\beta_{i}-\tau}\right)^{1-\tau},
\end{aligned}
$$




$$
\begin{aligned}
\Delta_{1}:= & \frac{T^{\alpha-\beta_{1}-\tau}}{\Gamma\left(\alpha-\beta_{1}\right)}\left(\frac{1-\tau}{\alpha-\beta_{1}-\tau}\right)^{1-\tau}\left\|l_{f}\right\|_{1 / \tau} \\
& +\sum_{i=1}^{2} \tilde{\rho}_{i}\left\|l_{f}\right\|_{1 / \tau}\left(\left|b_{i}\right| \frac{T^{\alpha-\beta_{i}-\tau}}{\Gamma\left(\alpha-\beta_{i}\right)}+\left|a_{i}\right| \frac{\eta^{\alpha-\beta_{i}-\tau}}{\Gamma\left(\alpha-\beta_{i}\right)}\right) \\
& \times\left(\frac{1-\tau}{\alpha-\beta_{i}-\tau}\right)^{1-\tau}, \\
\Delta_{2}:= & \frac{T^{\alpha-\beta_{2}-\tau}}{\Gamma\left(\alpha-\beta_{2}\right)}\left(\frac{1-\tau}{\alpha-\beta_{2}-\tau}\right)^{1-\tau}\left\|l_{f}\right\|_{1 / \tau} \\
& +\hat{\rho}_{2}\left\|l_{f}\right\|_{1 / \tau}\left(\left|b_{2}\right| \frac{T^{\alpha-\beta_{2}-\tau}}{\Gamma\left(\alpha-\beta_{2}\right)}+\left|a_{2}\right| \frac{\eta^{\alpha-\beta_{2}-\tau}}{\Gamma\left(\alpha-\beta_{2}\right)}\right) \\
& \times\left(\frac{1-\tau}{\alpha-\beta_{2}-\tau}\right)^{1-\tau} .
\end{aligned}
$$

\section{Theorem 7. Assume the following.}

$\left(\mathrm{H}_{1}\right)$ The function $f:[0, T] \times \mathbb{R} \times \mathbb{R} \times \mathbb{R} \rightarrow \mathbb{R}$ is jointly continuous.

$\left(\mathrm{H}_{2}\right)$ There exists a function $l_{f} \in L^{1 / \tau}\left([0, T] ; \mathbb{R}^{+}\right)$with $\tau \in$ $\left(0, \min \left(1, \alpha-\beta_{2}\right)\right)$ such that

$$
\begin{aligned}
& \left|f\left(t, u_{1}, u_{2}, u_{3}\right)-f\left(t, v_{1}, v_{2}, v_{3}\right)\right| \\
& \quad \leq l_{f}(t)\left(\left|u_{1}-v_{1}\right|+\left|u_{2}-v_{2}\right|+\left|u_{3}-v_{3}\right|\right)
\end{aligned}
$$

for each $\left(t, u_{1}, u_{2}, u_{3}\right),\left(t, v_{1}, v_{2}, v_{3}\right) \in[0, T] \times \mathbb{R} \times \mathbb{R} \times \mathbb{R}$.

$\left(\mathrm{H}_{3}\right)$ The function $g_{i}:[0, T] \times \mathbb{R} \rightarrow \mathbb{R}$ is jointly continuous and there exists $l_{g_{i}} \in L^{1}\left([0, T], \mathbb{R}^{+}\right)$such that

$\left|g_{i}(t, u)-g_{i}(t, v)\right| \leq l_{g_{i}}(t)|u-v|, \quad i=0,1,2$

for each $(t, u),(t, v) \in[0, T] \times \mathbb{R}$.

If

$$
\begin{aligned}
\left(\Delta_{0}\right. & \left.+\Delta_{1}+\Delta_{2}\right)\left\|l_{f}\right\|_{1 / \tau}+\sum_{i=0}^{2} \rho_{i}\left|\lambda_{i}\right|\left\|l_{g_{i}}\right\|_{1} \\
& +\sum_{i=1}^{2} \tilde{\rho}_{i}\left|\lambda_{i}\right|\left\|l_{g_{i}}\right\|_{1}+\widehat{\rho}_{2}\left|\lambda_{2}\right|\left\|l_{g_{2}}\right\|_{1}<1,
\end{aligned}
$$

then the problem (4)-(5) has a unique solution on $[0, T]$.
Proof. In order to transform the BVP (4)-(5) into a fixed point problem, we consider the operator $\mathfrak{F}: C_{\beta}([0, T] ; \mathbb{R}) \rightarrow$ $C_{\beta}([0, T] ; \mathbb{R})$ which is defined by

$(\mathfrak{w})(t)$

$$
\begin{aligned}
= & \int_{0}^{t} \frac{(t-s)^{\alpha-1}}{\Gamma(\alpha)} f\left(s, u(s), \mathfrak{D}_{0^{+}}^{\beta_{1}} u(s), \mathfrak{D}_{0^{+}}^{\beta_{2}} u(s)\right) d s \\
& +\sum_{i=0}^{2} \omega_{i}(t) b_{i} \\
& \times \int_{0}^{T} \frac{(T-s)^{\alpha-\beta_{i}-1}}{\Gamma\left(\alpha-\beta_{i}\right)} f\left(s, u(s), \mathfrak{D}_{0^{+}}^{\beta_{1}} u(s), \mathfrak{D}_{0^{+}}^{\beta_{2}} u(s)\right) d s \\
& +\sum_{i=1}^{2} \omega_{i}(t) a_{i} \\
& \times \int_{0}^{\eta} \frac{(\eta-s)^{\alpha-\beta_{i}-1}}{\Gamma\left(\alpha-\beta_{i}\right)} f\left(s, u(s), \mathfrak{D}_{0^{+}}^{\beta_{1}} u(s), \mathfrak{D}_{0^{+}}^{\beta_{2}} u(s)\right) d s \\
& -\sum_{i=0}^{2} \omega_{i}(t) \lambda_{i} \int_{0}^{T} g_{i}(s, u(s)) d s
\end{aligned}
$$

and take its $\beta_{1}$ th and $\beta_{2}$ th fractional derivative to get

$$
\begin{aligned}
& \mathfrak{D}_{0^{+}}^{\beta_{1}}(\mathfrak{F u} u(t) \\
& =\int_{0}^{t} \frac{(t-s)^{\alpha-\beta_{1}-1}}{\Gamma\left(\alpha-\beta_{1}\right)} f\left(s, u(s), \mathfrak{D}_{0^{+}}^{\beta_{1}} u(s), \mathfrak{D}_{0^{+}}^{\beta_{2}} u(s)\right) d s \\
& \quad+\sum_{i=1}^{2} \mathfrak{D}_{0^{+}}^{\beta_{1}} \omega_{i}(t) b_{i} \\
& \quad \times \int_{0}^{T} \frac{(T-s)^{\alpha-\beta_{i}-1}}{\Gamma\left(\alpha-\beta_{i}\right)} f\left(s, u(s), \mathfrak{D}_{0^{+}}^{\beta_{1}} u(s), \mathfrak{D}_{0^{+}}^{\beta_{2}} u(s)\right) d s \\
& \quad+\sum_{i=1}^{2} \mathfrak{D}_{0^{+}}^{\beta_{1}} \omega_{i}(t) a_{i} \\
& \quad \times \int_{0}^{\eta} \frac{(\eta-s)^{\alpha-\beta_{i}-1}}{\Gamma\left(\alpha-\beta_{i}\right)} f\left(s, u(s), \mathfrak{D}_{0^{+}}^{\beta_{1}} u(s), \mathfrak{D}_{0^{+}}^{\beta_{2}} u(s)\right) d s \\
& \quad-\sum_{i=1}^{2} \mathfrak{D}_{0^{+}}^{\beta_{1}} \omega_{i}(t) \lambda_{i} \int_{0}^{T} g_{i}(s, u(s)) d s,
\end{aligned}
$$




$$
\begin{aligned}
& \mathfrak{D}_{0^{+}}^{\beta_{2}}(\mathfrak{F} u)(t) \\
& =\int_{0}^{t} \frac{(t-s)^{\alpha-\beta_{2}-1}}{\Gamma\left(\alpha-\beta_{2}\right)} f\left(s, u(s), \mathfrak{D}_{0^{+}}^{\beta_{1}} u(s), \mathfrak{D}_{0^{+}}^{\beta_{2}} u(s)\right) d s \\
& \quad+\mathfrak{D}_{0^{+}}^{\beta_{2}} \omega_{2}(t) b_{2} \\
& \quad \times \int_{0}^{T} \frac{(T-s)^{\alpha-\beta_{2}-1}}{\Gamma\left(\alpha-\beta_{2}\right)} f\left(s, u(s), \mathfrak{D}_{0^{+}}^{\beta_{1}} u(s), \mathfrak{D}_{0^{+}}^{\beta_{2}} u(s)\right) d s \\
& \quad+\mathfrak{D}_{0^{+}}^{\beta_{2}} \omega_{2}(t) a_{2} \\
& \quad \times \int_{0}^{\eta} \frac{(\eta-s)^{\alpha-\beta_{2}-1}}{\Gamma\left(\alpha-\beta_{2}\right)} f\left(s, u(s), \mathfrak{D}_{0^{+}}^{\beta_{1}} u(s), \mathfrak{D}_{0^{+}}^{\beta_{2}} u(s)\right) d s \\
& \quad-\mathfrak{D}_{0^{+}}^{\beta_{2}} \omega_{2}(t) \lambda_{2} \int_{0}^{T} g_{2}(s, u(s)) d s .
\end{aligned}
$$

Clearly, due to $f, g_{0}, g_{1}$, and $g_{2}$ being jointly continuous, the expressions (32)-(33) are well defined. It is obvious that the fixed point of the operator $\mathfrak{F}$ is a solution of the problem (4)(5). To show existence and uniqueness of the solution (12)(13), we use the Banach fixed point theorem. To this end, we show that $\mathfrak{F}$ is contraction:

$$
\begin{aligned}
& |(\mathfrak{J u})(t)-(\mathfrak{F v})(t)| \\
& \leq \int_{0}^{t} \frac{(t-s)^{\alpha-1}}{\Gamma(\alpha)} \\
& \times \mid f\left(s, u(s), \mathfrak{D}_{0^{+}}^{\beta_{1}} u(s), \mathfrak{D}_{0^{+}}^{\beta_{2}} u(s)\right) \\
& -f\left(s, v(s), \mathfrak{D}_{0^{+}}^{\beta_{1}} v(s), \mathfrak{D}_{0^{+}}^{\beta_{2}} v(s)\right) \mid d s \\
& +\sum_{i=0}^{2}\left|\omega_{i}(t)\right|\left|b_{i}\right| \int_{0}^{T} \frac{(T-s)^{\alpha-\beta_{i}-1}}{\Gamma\left(\alpha-\beta_{i}\right)} \\
& \times \mid f\left(s, u(s), \mathfrak{D}_{0^{+}}^{\beta_{1}} u(s), \mathfrak{D}_{0^{+}}^{\beta_{2}} u(s)\right) \\
& -f\left(s, v(s), \mathfrak{D}_{0^{+}}^{\beta_{1}} v(s), \mathfrak{D}_{0^{+}}^{\beta_{2}} v(s)\right) \mid d s \\
& +\sum_{i=1}^{2}\left|\omega_{i}(t)\right|\left|a_{i}\right| \int_{0}^{\eta} \frac{(\eta-s)^{\alpha-\beta_{i}-1}}{\Gamma\left(\alpha-\beta_{i}\right)} \\
& \times \mid f\left(s, u(s), \mathfrak{D}_{0^{+}}^{\beta_{1}} u(s), \mathfrak{D}_{0^{+}}^{\beta_{2}} u(s)\right) \\
& -f\left(s, v(s), \mathfrak{D}_{0^{+}}^{\beta_{1}} v(s), \mathfrak{D}_{0^{+}}^{\beta_{2}} v(s)\right) \mid d s \\
& +\sum_{i=0}^{2}\left|\omega_{i}(t)\right|\left|\lambda_{i}\right| \int_{0}^{T}\left|g_{i}(s, u(s))-g_{i}(s, v(s))\right| d s
\end{aligned}
$$

$$
\begin{aligned}
& \leq\left\|l_{f}\right\|_{1 / \tau} \frac{T^{\alpha-\tau}}{\Gamma(\alpha)}\left(\frac{1-\tau}{\alpha-\tau}\right)^{1-\tau}\|u-v\|_{\beta} \\
& +\left(\left\|l_{f}\right\|_{1 / \tau} \sum_{i=0}^{2} \rho_{i}\left(\left|b_{i}\right| \frac{T^{\alpha-\beta_{i}-\tau}}{\Gamma\left(\alpha-\beta_{i}\right)}+\left|a_{i}\right| \frac{\eta^{\alpha-\beta_{i}-\tau}}{\Gamma\left(\alpha-\beta_{i}\right)}\right)\right. \\
& \left.\quad \times\left(\frac{1-\tau}{\alpha-\beta_{i}-\tau}\right)^{1-\tau}+\sum_{i=0}^{2} \rho_{i}\left|\lambda_{i}\right|\left\|l_{g_{i}}\right\|_{1}\right)\|u-v\|_{\beta} \\
& =\left(\Delta_{0}\left\|l_{f}\right\|_{1 / \tau}+\sum_{i=0}^{2} \rho_{i}\left|\lambda_{i}\right|\left\|l_{g_{i}}\right\|_{1}\right)\|u-v\|_{\beta} .
\end{aligned}
$$

On the other hand,

$$
\begin{aligned}
& \left|\mathfrak{D}_{0+}^{\beta_{1}}(\mathfrak{F} u)(t)-\mathfrak{D}_{0+}^{\beta_{1}}(\mathfrak{F} v)(t)\right| \\
& \leq \int_{0}^{t} \frac{(t-s)^{\alpha-\beta_{1}-1}}{\Gamma\left(\alpha-\beta_{1}\right)} \\
& \times \mid f\left(s, u(s), \mathfrak{D}_{0^{+}}^{\beta_{1}} u(s), \mathfrak{D}_{0^{+}}^{\beta_{2}} u(s)\right) \\
& -f\left(s, v(s), \mathfrak{D}_{0^{+}}^{\beta_{1}} v(s), \mathfrak{D}_{0^{+}}^{\beta_{2}} v(s)\right) \mid d s \\
& +\sum_{i=1}^{2}\left|\mathfrak{D}_{0+}^{\beta_{1}} \omega_{i}(t)\right|\left|b_{i}\right| \\
& \times \int_{0}^{T} \frac{(T-s)^{\alpha-\beta_{i}-1}}{\Gamma\left(\alpha-\beta_{i}\right)} \mid f\left(s, u(s), \mathfrak{D}_{0^{+}}^{\beta_{1}} u(s), \mathfrak{D}_{0^{+}}^{\beta_{2}} u(s)\right) \\
& -f\left(s, v(s), \mathfrak{D}_{0^{+}}^{\beta_{1}} v(s), \mathfrak{D}_{0^{+}}^{\beta_{2}} v(s)\right) \mid d s \\
& +\sum_{i=1}^{2}\left|\mathfrak{D}_{0+}^{\beta_{1}} \omega_{i}(t)\right|\left|a_{i}\right| \\
& \times \int_{0}^{\eta} \frac{(\eta-s)^{\alpha-\beta_{i}-1}}{\Gamma\left(\alpha-\beta_{i}\right)} \mid f\left(s, u(s), \mathfrak{D}_{0^{+}}^{\beta_{1}} u(s), \mathfrak{D}_{0^{+}}^{\beta_{2}} u(s)\right) \\
& -f\left(s, v(s), \mathfrak{D}_{0^{+}}^{\beta_{1}} v(s), \mathfrak{D}_{0^{+}}^{\beta_{2}} v(s)\right) \mid d s \\
& +\sum_{i=1}^{2}\left|\mathfrak{D}_{0+}^{\beta_{1}} \omega_{i}(t)\right|\left|\lambda_{i}\right| \int_{0}^{T}\left|g_{i}(s, u(s))-g_{i}(s, v(s))\right| d s \\
& \leq \frac{T^{\alpha-\beta_{1}-\tau}}{\Gamma\left(\alpha-\beta_{1}\right)}\left(\frac{1-\tau}{\alpha-\beta_{1}-\tau}\right)^{1-\tau}\left\|l_{f}\right\|_{1 / \tau}\|u-v\|_{\beta} \\
& +\sum_{i=1}^{2} \widetilde{\rho}_{i}\left[\left\|l_{f}\right\|_{1 / \tau}\left(\left|b_{i}\right| \frac{T^{\alpha-\beta_{i}-\tau}}{\Gamma\left(\alpha-\beta_{i}\right)}+\left|a_{i}\right| \frac{\eta^{\alpha-\beta_{i}-\tau}}{\Gamma\left(\alpha-\beta_{i}\right)}\right)\right. \\
& \left.\times\left(\frac{1-\tau}{\alpha-\beta_{i}-\tau}\right)^{1-\tau}+\left|\lambda_{i}\right|\left\|l_{g_{i}}\right\|_{1}\right]\|u-v\|_{\beta} \\
& =\left(\Delta_{1}\left\|l_{f}\right\|_{1 / \tau}+\sum_{i=1}^{2} \widetilde{\rho}_{i}\left|\lambda_{i}\right|\left\|l_{g_{i}}\right\|_{1}\right)\|u-v\|_{\beta} \text {. }
\end{aligned}
$$


Similarly,

$$
\begin{aligned}
& \left|\mathfrak{D}_{0+}^{\beta_{2}}(\mathfrak{F} u)(t)-\mathfrak{D}_{0+}^{\beta_{2}}(\mathfrak{F} v)(t)\right| \\
& \leq \int_{0}^{t} \frac{(t-s)^{\alpha-\beta_{2}-1}}{\Gamma\left(\alpha-\beta_{2}\right)} \\
& \times \mid f\left(s, u(s), \mathfrak{D}_{0^{+}}^{\beta_{1}} u(s), \mathfrak{D}_{0^{+}}^{\beta_{2}} u(s)\right) \\
& -f\left(s, v(s), \mathfrak{D}_{0^{+}}^{\beta_{1}} v(s), \mathfrak{D}_{0^{+}}^{\beta_{2}} v(s)\right) \mid d s \\
& +\left|\mathfrak{D}_{0+}^{\beta_{2}} \omega_{2}(t)\right|\left|b_{2}\right| \\
& \times \int_{0}^{T} \frac{(T-s)^{\alpha-\beta_{2}-1}}{\Gamma\left(\alpha-\beta_{2}\right)} \\
& \times \mid f\left(s, u(s), \mathfrak{D}_{0^{+}}^{\beta_{1}} u(s), \mathfrak{D}_{0^{+}}^{\beta_{2}} u(s)\right) \\
& -f\left(s, v(s), \mathfrak{D}_{0^{+}}^{\beta_{1}} v(s), \mathfrak{D}_{0^{+}}^{\beta_{2}} v(s)\right) \mid d s \\
& +\left|\mathfrak{P}_{0+}^{\beta_{2}} \omega_{2}(t)\right|\left|a_{2}\right| \\
& \times \int_{0}^{\eta} \frac{(\eta-s)^{\alpha-\beta_{2}-1}}{\Gamma\left(\alpha-\beta_{2}\right)} \\
& \times \mid f\left(s, u(s), \mathfrak{D}_{0^{+}}^{\beta_{1}} u(s), \mathfrak{D}_{0^{+}}^{\beta_{2}} u(s)\right) \\
& -f\left(s, v(s), \mathfrak{D}_{0^{+}}^{\beta_{1}} v(s), \mathfrak{D}_{0^{+}}^{\beta_{2}} v(s)\right) \mid d s \\
& +\left|\mathfrak{P}_{0+}^{\beta_{2}} \omega_{2}(t)\right|\left|\lambda_{2}\right| \\
& \times \int_{0}^{T}\left|g_{2}(s, u(s))-g_{2}(s, v(s))\right| d s \\
& \leq \frac{T^{\alpha-\beta_{2}-\tau}}{\Gamma\left(\alpha-\beta_{2}\right)}\left(\frac{1-\tau}{\alpha-\beta_{2}-\tau}\right)^{1-\tau}\left\|l_{f}\right\|_{1 / \tau}\|u-v\|_{\beta} \\
& +\left(\hat{\rho}_{2}\left(\left|b_{2}\right| \frac{T^{\alpha-\beta_{2}-\tau}}{\Gamma\left(\alpha-\beta_{2}\right)}+\left|a_{2}\right| \frac{\eta^{\alpha-\beta_{2}-\tau}}{\Gamma\left(\alpha-\beta_{2}\right)}\right)\right. \\
& \left.\times\left(\frac{1-\tau}{\alpha-\beta_{2}-\tau}\right)^{1-\tau}\left\|l_{f}\right\|_{1 / \tau}+\hat{\rho}_{2}\left|\lambda_{2}\right|\left\|l_{g_{2}}\right\|_{1}\right) \\
& \times\|u-v\|_{\beta} \\
& =\left(\Delta_{2}\left\|l_{f}\right\|_{1 / \tau}+\hat{\rho}_{2}\left|\lambda_{2}\right|\left\|l_{g_{2}}\right\|_{1}\right)\|u-v\|_{\beta} .
\end{aligned}
$$

Here, in estimations (34)-(36), we used the Hölder inequality:

$$
\begin{aligned}
& \int_{0}^{t} l_{f}(s)(t-s)^{\alpha-m-1} d s \\
& \leq\left(\int_{0}^{t}\left(l_{f}(s)\right)^{1 / \tau} d s\right)^{\tau}\left(\int_{0}^{t}\left((t-s)^{\alpha-m-1}\right)^{1 /(1-\tau)} d s\right)^{1-\tau}
\end{aligned}
$$

$$
=\left\|l_{f}\right\|_{L^{1 / \tau}}\left(\frac{1-\tau}{\alpha-m-\tau}\right)^{1-\tau} t^{\alpha-m-\tau}, \quad \text { if } 0<\tau<\min (1, \alpha-m) .
$$

From (34)-(36), it follows that

$$
\begin{aligned}
& \|(\mathfrak{F u})-(\mathfrak{F} v)\|_{\beta} \\
& \leq\left[\left(\Delta_{0}+\Delta_{1}+\Delta_{2}\right)\left\|l_{f}\right\|_{1 / \tau}+\sum_{i=0}^{2} \rho_{i}\left|\lambda_{i}\right|\left\|l_{g_{i}}\right\|_{1}\right. \\
& \left.\quad+\sum_{i=1}^{2} \widetilde{\rho}_{i}\left|\lambda_{i}\right|\left\|l_{g_{i}}\right\|_{1}+\hat{\rho}_{2}\left|\lambda_{2}\right|\left\|l_{g_{2}}\right\|_{1}\right]\|u-v\|_{\beta} .
\end{aligned}
$$

Consequently, by (31), $\mathfrak{F}$ is a contraction mapping. As a consequence of the Banach fixed point theorem, we deduce that $\mathfrak{F}$ has a fixed point which is a solution of the problem (4)-(5).

Remark 8. In the assumptions $\left(\mathrm{H}_{2}\right)$, if $l_{f}$ is a positive constant, then the condition (31) can be replaced by

$$
\begin{aligned}
& \frac{l_{f} T^{\alpha}}{\Gamma(\alpha+1)} \\
& \quad+l_{f} \sum_{i=0}^{2} \rho_{i}\left(\left|b_{i}\right| \frac{T^{\alpha-\beta_{i}}}{\Gamma\left(\alpha-\beta_{i}+1\right)}+\left|a_{i}\right| \frac{\eta^{\alpha-\beta_{i}}}{\Gamma\left(\alpha-\beta_{i}+1\right)}\right) \\
& +\frac{l_{f} T^{\alpha-\beta_{1}}}{\Gamma\left(\alpha-\beta_{1}+1\right)} \\
& +l_{f} \sum_{i=1}^{2} \tilde{\rho}_{i}\left(\left|b_{i}\right| \frac{T^{\alpha-\beta_{i}}}{\Gamma\left(\alpha-\beta_{i}+1\right)}+\left|a_{i}\right| \frac{\eta^{\alpha-\beta_{i}}}{\Gamma\left(\alpha-\beta_{i}+1\right)}\right) \\
& +\frac{l_{f} T^{\alpha-\beta_{2}}}{\Gamma\left(\alpha-\beta_{2}+1\right)} \\
& +l_{f} \widehat{\rho}_{2}\left(\left|b_{2}\right| \frac{T^{\alpha-\beta_{2}}}{\Gamma\left(\alpha-\beta_{2}+1\right)}+\left|a_{2}\right| \frac{\eta^{\alpha-\beta_{2}}}{\Gamma\left(\alpha-\beta_{2}+1\right)}\right) \\
& +\sum_{i=0}^{2} \rho_{i}\left|\lambda_{i}\right|\left\|l_{g_{i}}\right\|_{1}+\sum_{i=1}^{2} \tilde{\rho}_{i}\left|\lambda_{i}\right|\left\|l_{g_{i}}\right\|_{1} \\
& +\widehat{\rho}_{2}\left|\lambda_{2}\right|\left\|l_{g_{2}}\right\|_{1}<1 .
\end{aligned}
$$

\section{Existence Results}

To prove the existence of solutions for BVP (4)-(5), we recall the following known nonlinear alternative.

Theorem 9 (nonlinear alternative). Let $X$ be a Banach space; let $B$ be a closed, convex subset of $X$; let $W$ be an open subset of $B$ and $0 \in W$. Suppose that $F: \bar{W} \rightarrow B$ is a continuous and compact map. Then, either (a) F has a fixed point in $\bar{W}$ or (b) there exist an $x \in \partial W$ (the boundary of $W$ ) and $\lambda \in(0,1)$ with $x=\lambda F(x)$. 
Theorem 10. Assume that

$\left(\mathrm{H}_{4}\right)$ functions $f:[0, T] \times \mathbb{R} \times \mathbb{R} \times \mathbb{R} \rightarrow \mathbb{R}, g_{i}:[0, T] \times \mathbb{R} \rightarrow$ $\mathbb{R}(i=0,1,2)$ are jointly continuous;

$\left(\mathrm{H}_{5}\right)$ there exist nondecreasing functions $\varphi:[0, \infty) \rightarrow$ $[0, \infty), \psi_{i}:[0, \infty) \rightarrow[0, \infty)$ and functions $l_{f} \in L^{1 / \tau}\left([0, T], \mathbb{R}^{+}\right), l_{g_{i}} \in L^{1}\left([0, T], \mathbb{R}^{+}\right)$with $\tau \in$ $\left(0, \min \left(1, \alpha-\beta_{2}\right)\right)$ such that

$$
\begin{gathered}
|f(t, u, v, w)| \leq l_{f}(t) \varphi(|u|+|v|+|w|), \\
\left|g_{i}(t, u)\right| \leq l_{g_{i}}(t) \psi_{i}(|u|),
\end{gathered}
$$

$i=0,1,2$, for all $t \in[0, T]$ and $u, v, w \in \mathbb{R}$;

$\left(\mathrm{H}_{6}\right)$ there exists a constant $K>0$ such that

$$
\begin{aligned}
& K\left(\varphi(K)\left\|l_{f}\right\|_{1 / \tau}\left(\Delta_{0}+\Delta_{1}+\Delta_{2}\right)\right. \\
& \left.\quad+\sum_{i=0}^{2}\left(\rho_{i}+\tilde{\rho}_{i}+\hat{\rho}_{i}\right)\left|\lambda_{i}\right| \psi_{i}(K)\left\|l_{g_{i}}\right\|_{1}\right)^{-1}>1 .
\end{aligned}
$$

Then the problem (4)-(5) has at least one solution on $[0, T]$.

Proof. Let $B_{r}:=\left\{u \in C_{\beta}([0, T] ; \mathbb{R}):\|u\|_{\beta} \leq r\right\}$.

Step 1. We show that the operator $\mathfrak{F}: C_{\beta}([0, T] ; \mathbb{R}) \rightarrow$ $C_{\beta}([0, T] ; \mathbb{R})$ defined by $(32)$ maps $B_{r}$ into bounded set.

For each $u \in B_{r}$, we have

$$
\begin{aligned}
& \mid(\mathfrak{F u}(t) \mid \\
& \leq \frac{\varphi(r)}{\Gamma(\alpha)} \int_{0}^{t}(t-s)^{\alpha-1}\left|l_{f}(s)\right| d s \\
& \quad+\varphi(r) \sum_{i=0}^{2} \rho_{i}\left|b_{i}\right| \frac{1}{\Gamma\left(\alpha-\beta_{i}\right)} \int_{0}^{T}(T-s)^{\alpha-\beta_{i}-1}\left|l_{f}(s)\right| d s \\
& \quad+\varphi(r) \sum_{i=1}^{2} \rho_{i}\left|a_{i}\right| \frac{1}{\Gamma\left(\alpha-\beta_{i}\right)} \int_{0}^{\eta}(\eta-s)^{\alpha-\beta_{i}-1}\left|l_{f}(s)\right| d s \\
& \quad+\sum_{i=0}^{2} \rho_{i}\left|\lambda_{i}\right| \psi_{i}(r) \int_{0}^{T}\left|l_{g_{i}}(s)\right| d s .
\end{aligned}
$$

By the Hölder inequality, we have

$$
\begin{aligned}
& |(\mathfrak{J u})(t)| \\
& \leq \varphi(r)\left\|l_{f}\right\|_{1 / \tau}\left(\frac{T^{\alpha-\tau}}{\Gamma(\alpha)}\left(\frac{1-\tau}{\alpha-\tau}\right)^{1-\tau}\right. \\
& +\sum_{i=0}^{2} \rho_{i}\left|b_{i}\right| \frac{T^{\alpha-\beta_{i}-\tau}}{\Gamma\left(\alpha-\beta_{i}\right)}\left(\frac{1-\tau}{\alpha-\beta_{i}-\tau}\right)^{1-\tau} \\
& \left.+\sum_{i=1}^{2} \rho_{i}\left|a_{i}\right| \frac{\eta^{\alpha-\beta_{i}-\tau}}{\Gamma\left(\alpha-\beta_{i}\right)}\left(\frac{1-\tau}{\alpha-\beta_{i}-\tau}\right)^{1-\tau}\right) \\
& +\sum_{i=0}^{2} \rho_{i}\left|\lambda_{i}\right| \psi_{i}(r)\left\|l_{g_{i}}\right\|_{1} \\
& =\varphi(r)\left\|l_{f}\right\|_{1 / \tau} \Delta_{0}+\sum_{i=0}^{2} \rho_{i}\left|\lambda_{i}\right| \psi_{i}(r)\left\|l_{g_{i}}\right\|_{1} .
\end{aligned}
$$

In a similar manner,

$$
\begin{aligned}
& \left|\mathfrak{D}_{0+}^{\beta_{1}}(\mathfrak{F u})(t)\right| \\
& \leq \varphi(r)\left\|l_{f}\right\|_{1 / \tau}\left(\frac{T^{\alpha-\beta_{1}-\tau}}{\Gamma\left(\alpha-\beta_{1}\right)}\left(\frac{1-\tau}{\alpha-\beta_{1}-\tau}\right)^{1-\tau}\right. \\
& +\sum_{i=1}^{2} \tilde{\rho}_{i}\left|b_{i}\right| \frac{T^{\alpha-\beta_{i}-\tau}}{\Gamma\left(\alpha-\beta_{i}\right)}\left(\frac{1-\tau}{\alpha-\beta_{i}-\tau}\right)^{1-\tau} \\
& \left.+\sum_{i=1}^{2} \tilde{\rho}_{i}\left|a_{i}\right| \frac{\eta^{\alpha-\beta_{i}-\tau}}{\Gamma\left(\alpha-\beta_{i}\right)}\left(\frac{1-\tau}{\alpha-\beta_{i}-\tau}\right)^{1-\tau}\right) \\
& +\sum_{i=1}^{2} \widetilde{\rho}_{i}\left|\lambda_{i}\right| \psi_{i}(r)\left\|l_{g_{i}}\right\|_{1} \\
& =\varphi(r)\left\|l_{f}\right\|_{1 / \tau} \Delta_{1}+\sum_{i=1}^{2} \widetilde{\rho}_{i}\left|\lambda_{i}\right| \psi_{i}(r)\left\|l_{g_{i}}\right\|_{1}, \\
& \left|\mathfrak{D}_{0+}^{\beta_{2}}(\mathfrak{F u})(t)\right| \\
& \leq \varphi(r)\left\|l_{f}\right\|_{1 / \tau}\left(\frac{T^{\alpha-\beta_{2}-\tau}}{\Gamma\left(\alpha-\beta_{2}\right)}\left(\frac{1-\tau}{\alpha-\beta_{2}-\tau}\right)^{1-\tau}\right. \\
& +\widehat{\rho}_{2} \frac{T^{\alpha-\beta_{2}-\tau}\left|b_{2}\right|}{\Gamma\left(\alpha-\beta_{2}\right)}\left(\frac{1-\tau}{\alpha-\beta_{2}-\tau}\right)^{1-\tau} \\
& \left.+\widehat{\rho}_{2} \frac{\eta^{\alpha-\beta_{2}-\tau}\left|b_{2}\right|}{\Gamma\left(\alpha-\beta_{2}\right)}\left(\frac{1-\tau}{\alpha-\beta_{2}-\tau}\right)^{1-\tau}\right) \\
& +\widehat{\rho}_{2}\left|\lambda_{2}\right| \psi_{2}(r)\left\|l_{g_{2}}\right\|_{1} \\
& =\varphi(r)\left\|l_{f}\right\|_{1 / \tau} \Delta_{2}+\hat{\rho}_{2}\left|\lambda_{2}\right| \psi_{2}(r)\left\|l_{g_{2}}\right\|_{1} .
\end{aligned}
$$


Thus,

$$
\begin{aligned}
\|(\mathfrak{F u})\|_{\beta} \leq & \varphi(r)\left\|l_{f}\right\|_{1 / \tau}\left(\Delta_{0}+\Delta_{1}+\Delta_{2}\right) \\
& +\sum_{i=0}^{2}\left(\rho_{i}+\widetilde{\rho}_{i}+\widehat{\rho}_{i}\right)\left|\lambda_{i}\right| \psi_{i}(r)\left\|l_{g_{i}}\right\|_{1}
\end{aligned}
$$

Step 2. The families $\left\{\mathfrak{F} u: u \in B_{r}\right\},\left\{\mathfrak{D}_{0+}^{\beta_{1}}(\mathfrak{F} u): u \in B_{r}\right\}$, and $\left\{\mathfrak{D}_{0+}^{\beta_{2}}(\mathfrak{\Im} u): u \in B_{r}\right\}$ are equicontinuous.

Because of the continuity of $\omega_{i}(t)$ and assumption $\left(\mathrm{H}_{5}\right)$ we have

$$
\begin{aligned}
& \left|(\mathfrak{F u})\left(t_{2}\right)-(\mathfrak{F u})\left(t_{1}\right)\right| \\
& \leq \frac{1}{\Gamma(\alpha)} \varphi(r) \int_{t_{1}}^{t_{2}}\left(t_{2}-s\right)^{\alpha-1} l_{f}(s) d s \\
& \quad+\frac{1}{\Gamma(\alpha)} \varphi(r) \int_{0}^{t_{1}}\left(\left(t_{2}-s\right)^{\alpha-1}-\left(t_{1}-s\right)^{\alpha-1}\right) l_{f}(s) d s \\
& \quad+\varphi(r) \sum_{i=0}^{2}\left|\omega_{i}\left(t_{2}\right)-\omega_{i}\left(t_{1}\right)\right|\left|b_{i}\right| \\
& \quad \times \int_{0}^{T} \frac{(T-s)^{\alpha-\beta_{i}-1}}{\Gamma\left(\alpha-\beta_{i}\right)} l_{f}(s) d s \\
& \quad+\varphi(r) \sum_{i=1}^{2}\left|\omega_{i}\left(t_{2}\right)-\omega_{i}\left(t_{1}\right)\right|\left|a_{i}\right| \\
& \quad \times \int_{0}^{\eta} \frac{(\eta-s)^{\alpha-\beta_{i}-1}}{\Gamma\left(\alpha-\beta_{i}\right)} l_{f}(s) d s \\
& \quad+\sum_{i=0}^{2}\left|\omega_{i}\left(t_{2}\right)-\omega_{i}\left(t_{1}\right)\right|\left|\lambda_{i}\right| \psi_{i}(r)\left\|l_{g_{i}}\right\|_{1} \longrightarrow 0
\end{aligned}
$$

Therefore, $\left\{\mathfrak{F} u: u \in B_{r}\right\}$ is equicontinuous. Similarly, we may prove that $\left\{\mathfrak{D}_{0+}^{\beta_{1}}(\mathfrak{F} u): u \in B_{r}\right\}$ and $\left\{\mathfrak{D}_{0+}^{\beta_{2}}(\mathfrak{F} u): u \in B_{r}\right\}$ are equicontinuous.

Hence, by the Arzela-Ascoli theorem, the sets $\{\mathfrak{F} u: u \in$ $\left.B_{r}\right\},\left\{\mathfrak{D}_{0+}^{\beta_{1}}(\mathfrak{F u}): u \in B_{r}\right\}$, and $\left\{\mathfrak{D}_{0+}^{\beta_{2}}(\mathfrak{F} u): u \in B_{r}\right\}$ are relatively compact in $C([0, T] ; \mathbb{R})$. Therefore, $\mathfrak{F}\left(B_{r}\right)$ is a relatively compact subset of $C_{\beta}([0, T] ; \mathbb{R})$. Consequently, the operator $\mathfrak{F}$ is compact.

Step 3. $\mathfrak{F}$ has a fixed point in $W=\left\{u \in C_{\beta}([0, T] ; \mathbb{R}):\|u\|_{\beta}<\right.$ $K\}$.

We let $u=\lambda(\mathfrak{F} u)$ for $0<\lambda<1$. Then, for each $t \in[0, T]$,

$$
\begin{aligned}
\|u\|_{\beta}= & \|\lambda(\mathfrak{\Im} u)\|_{\beta} \leq \varphi\left(\|u\|_{\beta}\right)\left\|l_{f}\right\|_{1 / \tau}\left(\Delta_{0}+\Delta_{1}+\Delta_{2}\right) \\
& +\sum_{i=0}^{2}\left(\rho_{i}+\widetilde{\rho}_{i}+\widehat{\rho}_{i}\right)\left|\lambda_{i}\right| \psi_{i}\left(\|u\|_{\beta}\right)\left\|l_{g_{i}}\right\|_{1}
\end{aligned}
$$

In other words,

$$
\begin{aligned}
\|u\|_{\beta}( & \varphi\left(\|u\|_{\beta}\right)\left\|l_{f}\right\|_{1 / \tau}\left(\Delta_{0}+\Delta_{1}+\Delta_{2}\right) \\
& \left.+\sum_{i=0}^{2}\left(\rho_{i}+\tilde{\rho}_{i}+\hat{\rho}_{i}\right)\left|\lambda_{i}\right| \psi_{i}\left(\|u\|_{\beta}\right)\left\|l_{g_{i}}\right\|_{1}\right)^{-1} \leq 1 .
\end{aligned}
$$

According to the assumption $\left(\mathrm{H}_{6}\right)$, we know that there exists $K>0$ such that $K>\|u\|_{\beta}$ and

$$
\begin{aligned}
& K\left(\varphi(K)\left\|l_{f}\right\|_{1 / \tau}\left(\Delta_{0}+\Delta_{1}+\Delta_{2}\right)\right. \\
& \left.\quad+\sum_{i=0}^{2}\left(\rho_{i}+\widetilde{\rho}_{i}+\widehat{\rho}_{i}\right)\left|\lambda_{i}\right| \psi_{i}(K)\left\|l_{g_{i}}\right\|_{1}\right)^{-1}>1 .
\end{aligned}
$$

In other words, for all $u \in \partial W$, we have $u \neq \lambda(\mathfrak{F} u)$. Since the operator $\mathfrak{F}: \bar{W} \rightarrow C_{\beta}([0, T] ; \mathbb{R})$ is continuous and compact, from Theorem 9 (a), we can deduce that $\mathfrak{F}$ has a fixed point in $\bar{W}$.

Remark 11. Notice that analogues of Theorems 7 and 10 for the case $f(t, u, v, w)=f(t, u)$ were considered in [9]. Thus, our results are a generalization of [9] in the special case when (fractional) differential inclusion is replaced by (fractional) differential equation.

Remark 12. Since the number $\left(\alpha-\beta_{2}-1\right)$ can be negative, the function $(T-s)^{\alpha-\beta_{2}-1} \notin L^{\infty}([0, T], \mathbb{R})$. That is why in Theorems 7 and 10 it is assumed that $l_{f} \in L^{1 / \tau}, \tau \in$ $\left(0, \min \left(1, \alpha-\beta_{2}\right)\right)$.

\section{Examples}

Example 1. Consider the following boundary value problem of fractional differential equation:

$$
\begin{aligned}
& \mathfrak{D}_{0^{+}}^{5 / 2} u(t) \\
& =\frac{1}{11}\left(\frac{|u(t)|}{1+|u(t)|}+\frac{\left|\mathfrak{D}_{0^{+}}^{1 / 2} u(t)\right|}{1+\left|\mathfrak{D}_{0^{+}}^{1 / 2} u(t)\right|}+\tan ^{-1}\left(\mathfrak{D}_{0^{+}}^{3 / 2} u(t)\right)\right) \\
& u(0)+u(1)=\int_{0}^{1} \frac{u(s)}{(1+s)^{2}} d s, \quad 0 \leq 1, \\
& \mathfrak{D}_{0^{+}}^{1 / 2} u\left(\frac{1}{10}\right)+\mathfrak{D}_{0^{+}}^{1 / 2} u(1)=\frac{1}{2} \int_{0}^{1}\left(\frac{e^{s} u(s)}{1+2 e^{s}}+\frac{1}{2}\right) d s, \\
& \mathfrak{D}_{0^{+}}^{3 / 2} u\left(\frac{1}{10}\right)+\mathfrak{D}_{0^{+}}^{3 / 2} u(1)=\frac{1}{3} \int_{0}^{1}\left(\frac{u(s)}{1+e^{s}}+\frac{3}{4}\right) d s .
\end{aligned}
$$


Here,

$$
\begin{gathered}
\alpha=\frac{5}{2}, \quad \beta_{1}=\frac{1}{2}, \quad \beta_{2}=\frac{3}{2}, \\
T=1, \quad a_{0}=b_{0}=a_{1}=b_{1}=a_{2}=b_{2}=1, \\
\eta=\frac{1}{10}, \quad \lambda_{0}=1, \quad \lambda_{1}=\frac{1}{2}, \\
\lambda_{2}=\frac{1}{3}, \quad l_{g_{0}}=l_{g_{1}}=l_{g_{2}}=1, \\
f(t, u, v, w):=\frac{1}{11}\left(\frac{u}{1+u}+\frac{v}{1+v}+\tan ^{-1}(w)\right), \\
\left.g_{0}(t, u):=\frac{u}{(1+t)^{2}}, \quad g_{f}(t)=\frac{1}{11}, u\right):=\frac{e^{t} u}{1+2 e^{t}}+\frac{1}{2}, \\
g_{2}(t, u):=\frac{u}{1+e^{t}}+\frac{3}{4} .
\end{gathered}
$$

Since $1.77<\Gamma(1 / 2)<1.78,0.88<\Gamma(3 / 2)<0.89,1.32<$ $\Gamma(5 / 2)<1.33$, and $3.32<\Gamma(7 / 2)<3.33$, with simple calculations, we show that

$$
\begin{array}{ccc}
\Delta_{0}=2.34, & \Delta_{1}=0.19, & \Delta_{2}=0.15, \\
\rho_{0}=0.5, \quad \rho_{1}=1.01, & \rho_{2}=1.2, \\
\tilde{\rho}_{0}=0, & \tilde{\rho}_{1}=0.76, \quad \tilde{\rho}_{2}=0.9, \\
\hat{\rho}_{0}=\hat{\rho}_{1}=0, \quad \hat{\rho}_{2}=0.51 .
\end{array}
$$

Furthermore,

$$
\begin{aligned}
\left(\Delta_{0}+\Delta_{1}+\Delta_{2}\right)\left\|l_{f}\right\|_{1 / \tau}+\sum_{i=0}^{2} \rho_{i}\left|\lambda_{i}\right|\left\|l_{g_{i}}\right\|_{1} \\
\\
+\sum_{i=1}^{2} \tilde{\rho}_{i}\left|\lambda_{i}\right|\left\|l_{g_{i}}\right\|_{1}+\widehat{\rho}_{2}\left|\lambda_{2}\right|\left\|l_{g_{2}}\right\|_{1}<2.7 \frac{1}{11}+0.75<1 .
\end{aligned}
$$

Thus, all the assumptions of Theorem 7 are satisfied. Hence, the problem (50) has a unique solution on $[0,1]$.
Example 2. Consider the following boundary value problem of fractional differential equation:

$$
\begin{gathered}
\mathfrak{D}_{0^{+}}^{5 / 2} u(t)=\frac{|u(t)|^{3}}{9\left(|u(t)|^{3}+3\right)}+\frac{\left|\sin \mathfrak{D}_{0^{+}}^{1 / 2} u(t)\right|}{9\left(\left|\sin \mathfrak{D}_{0^{+}}^{1 / 2} u(t)\right|+1\right)}+\frac{1}{12}, \\
t \in[0,1] \\
u(0)+u(1)=\int_{0}^{1} \frac{u(s)}{3(1+s)^{2}} d s \\
\mathfrak{D}_{0^{+}}^{1 / 2} u\left(\frac{1}{10}\right)+\mathfrak{D}_{0^{+}}^{1 / 2} u(1)=\frac{1}{2} \int_{0}^{1} \frac{e^{s} u(s)}{3\left(1+e^{s}\right)^{2}} d s \\
\mathfrak{D}_{0^{+}}^{3 / 2} u\left(\frac{1}{10}\right)+\mathfrak{D}_{0^{+}}^{3 / 2} u(1)=\frac{1}{3} \int_{0}^{1} \frac{u(s)}{3\left(1+e^{s}\right)^{2}} d s
\end{gathered}
$$

where $f$ is given by

$$
f(t, u, v, w)=\frac{|u|^{3}}{10\left(|u|^{3}+3\right)}+\frac{|\sin v|}{9(|\sin v|+1)}+\frac{1}{12}
$$

We have

$$
\begin{array}{r}
|f(t, u, v, w)| \leq \frac{|u|^{3}}{9\left(|u|^{3}+3\right)}+\frac{|\sin v|}{9(|\sin v|+1)}+\frac{1}{12} \leq \frac{11}{36} \\
u, v, w \in \mathbb{R} .
\end{array}
$$

Thus,

$$
\begin{aligned}
& |f(t, u, v, w)| \leq \frac{11}{36}=l_{f}(t) \varphi(|u|+|v|+|w|), \\
& \text { with } l_{f}(t)=\frac{1}{3}, \quad \varphi(t)=\frac{11}{12} .
\end{aligned}
$$

Moreover,

$$
\begin{aligned}
\alpha=\frac{5}{2}, & \beta_{1}=\frac{1}{2}, \quad \beta_{2}=\frac{3}{2}, \\
T=1, \quad \tau=\frac{1}{10}, & a_{0}=b_{0}=a_{1}=b_{1}=a_{2}=b_{2}=1, \\
\eta=\frac{1}{10}, & \lambda_{0}=1, \quad \lambda_{1}=\frac{1}{2},
\end{aligned}
$$




$$
\begin{gathered}
\lambda_{2}=\frac{1}{3}, \quad l_{g_{0}}=l_{g_{1}}=l_{g_{2}}=\frac{1}{3}, \\
\Delta_{0}=2.34, \quad \Delta_{1}=0.19, \quad \Delta_{2}=0.15, \\
\rho_{0}=0.5, \quad \rho_{1}=1.01, \quad \rho_{2}=1.2, \\
\tilde{\rho}_{0}=0, \quad \tilde{\rho}_{1}=0.76, \quad \tilde{\rho}_{2}=0.9, \\
\widehat{\rho}_{0}=\widehat{\rho}_{1}=0, \quad \hat{\rho}_{2}=0.51, \\
g_{0}(t, u):=\frac{u}{3(1+t)^{2}}, \\
g_{1}(t, u):=\frac{e^{t} u}{3\left(1+e^{t}\right)^{2}}, \\
g_{2}(t, u):=\frac{u}{3\left(1+e^{t}\right)^{2}}, \\
\psi_{i}(u)=u, \quad i=0,1,2 .
\end{gathered}
$$

From the condition

$$
\begin{aligned}
& K\left(\varphi(K)\left\|l_{f}\right\|_{1 / \tau}\left(\Delta_{0}+\Delta_{1}+\Delta_{2}\right)\right. \\
& \left.\quad+\sum_{i=0}^{2}\left(\rho_{i}+\tilde{\rho}_{i}+\hat{\rho}_{i}\right)\left|\lambda_{i}\right| \psi_{i}(K)\left\|l_{g_{i}}\right\|_{1}\right)^{-1}>1,
\end{aligned}
$$

we find that

$$
K>9.8 .
$$

Thus, all the conditions of Theorem 10 are satisfied. So, there exists at least one solution of problem $(54)$ on $[0,1]$.

\section{Conflict of Interests}

The authors declare that there is no conflict of interests regarding the publication of this paper.

\section{Acknowledgment}

The authors would like to thank the reviewers for their constructive suggestions and helpful comments.

\section{References}

[1] R. P. Agarwal, M. Benchohra, and S. Hamani, "A survey on existence results for boundary value problems of nonlinear fractional differential equations and inclusions," Acta Applicandae Mathematicae, vol. 109, no. 3, pp. 973-1033, 2010.

[2] R. P. Agarwal, D. O'Regan, and S. Stanek, "Positive solutions for mixed problems of singular fractional differential equations," Mathematische Nachrichten, vol. 285, no. 1, pp. 27-41, 2012.

[3] B. Ahmad and J. J. Nieto, "Riemann-Liouville fractional integrodifferential equations with fractional nonlocal integral boundary conditions," Boundary Value Problems, vol. 2011, article 36, 2011.
[4] B. Ahmad, J. J. Nieto, and A. Alsaedi, "Existence and uniqueness of solutions for nonlinear fractional differential equations with non-separated type integral boundary conditions," Acta Mathematica Scientia B, vol. 31, no. 6, pp. 2122-2130, 2011.

[5] B. Ahmad and S. K. Ntouyas, "Nonlinear fractional differential equations and inclusions of arbitrary order and multi-strip boundary conditions," Electronic Journal of Differential Equations, vol. 2012, article 98, 2012.

[6] B. Ahmad and S. K. Ntouyas, "Existence results for nonlocal boundary value problems of fractional differential equations and inclusions with strip conditions," Boundary Value Problems, vol. 2012, article 55, 21 pages, 2012.

[7] B. Ahmad and J. J. Nieto, "Existence of solutions for nonlocal boundary value problems of higher-order nonlinear fractional differential equations," Abstract and Applied Analysis, vol. 2009, Article ID 494720, 9 pages, 2009.

[8] B. Ahmad and S. K. Ntouyas, "A boundary value problem of fractional differential equations with anti-periodic type integral boundary conditions," Journal of Computational Analysis and Applications, vol. 15, no. 8, pp. 1372-1380, 2013.

[9] B. Ahmad, S. K. Ntouyas, and A. Alsaedi, "On fractional differential inclusions with anti-periodic type integral boundary conditions," Boundary Value Problems, vol. 2013, article 82, 2013.

[10] M. Aitalioubrahim, "Neumann boundary-value problems for differential inclusions in Banach spaces," Electronic Journal of Differential Equations, vol. 2010, no. 104, pp. 1-5, 2010.

[11] Z. Bai, "On positive solutions of a nonlocal fractional boundary value problem," Nonlinear Analysis: Theory, Methods \& Applications, vol. 72, no. 2, pp. 916-924, 2010.

[12] Z. Bai and W. Sun, "Existence and multiplicity of positive solutions for singular fractional boundary value problems," Computers \& Mathematics with Applications, vol. 63, no. 9, pp. 1369-1381, 2012.

[13] D. BĂleanu and O. G. Mustafa, "On the global existence of solutions to a class of fractional differential equations," Computers \& Mathematics with Applications, vol. 59, no. 5, pp. 1835-1841, 2010.

[14] M. El-Shahed, "Positive solutions for boundary value problem of nonlinear fractional differential equation," Abstract and Applied Analysis, vol. 2007, Article ID 10368, 8 pages, 2007.

[15] E. Hernandez, D. O'Regan, and K. Balachandran, "On recent developments in the theory of abstract differential equations with fractional derivatives," Nonlinear Analysis: Theory, Methods and Applications, vol. 73, no. 10, pp. 3462-3471, 2010.

[16] M. u. Rehman, R. A. Khan, and N. A. Asif, "Three point boundary value problems for nonlinear fractional differential equations," Acta Mathematica Scientia B, vol. 31, no. 4, pp. 13371346, 2011.

[17] X. Su and S. Zhang, "Solutions to boundary-value problems for nonlinear differential equations of fractional order," Electronic Journal of Differential Equations, no. 26, pp. 1-15, 2009.

[18] Y. Wang, L. Liu, and Y. Wu, "Positive solutions for a nonlocal fractional differential equation," Nonlinear Analysis: Theory, Methods \& Applications, vol. 74, no. 11, pp. 3599-3605, 2011.

[19] G. Wang, R. P. Agarwal, and A. Cabada, "Existence results and the monotone iterative technique for systems of nonlinear fractional differential equations," Applied Mathematics Letters, vol. 25, no. 6, pp. 1019-1024, 2012.

[20] S. G. Samko, A. A. Kilbas, and O. I. Marichev, Fractional Integrals and Derivatives: Theory and Applications, Gordon \& Breach, Yverdon, Switzerland, 1993. 
[21] I. Podlubny, Fractional Differential Equations, Academic Press, San Diego, Calif, USA, 1999.

[22] A. A. Kilbas, H. M. Srivastava, and J. J. Trujillo, Theory and Applications of Fractional Differential Equations, vol. 204 of North-Holland Mathematics Studies, Elsevier, Amsterdam, The Netherlands, 2006. 


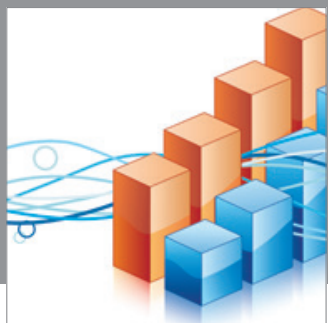

Advances in

Operations Research

mansans

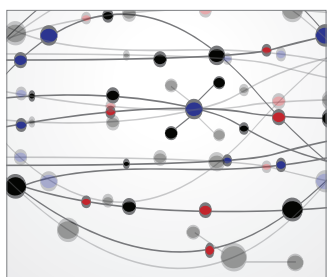

The Scientific World Journal
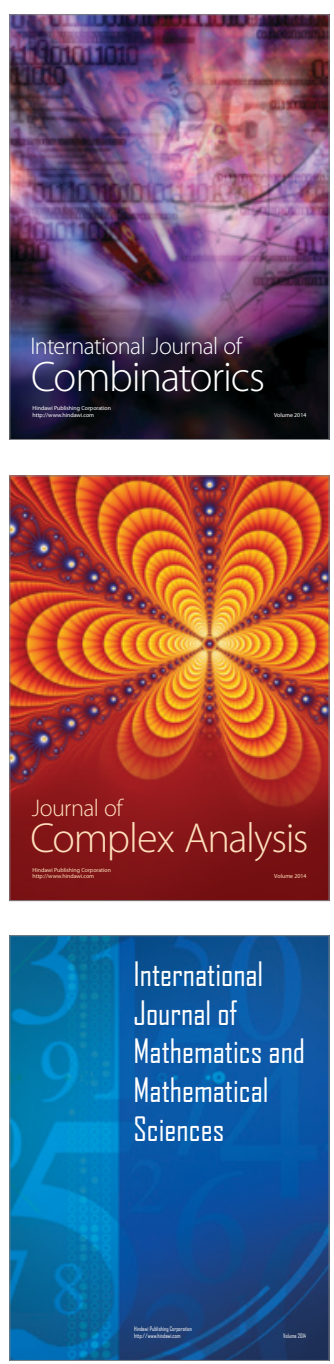
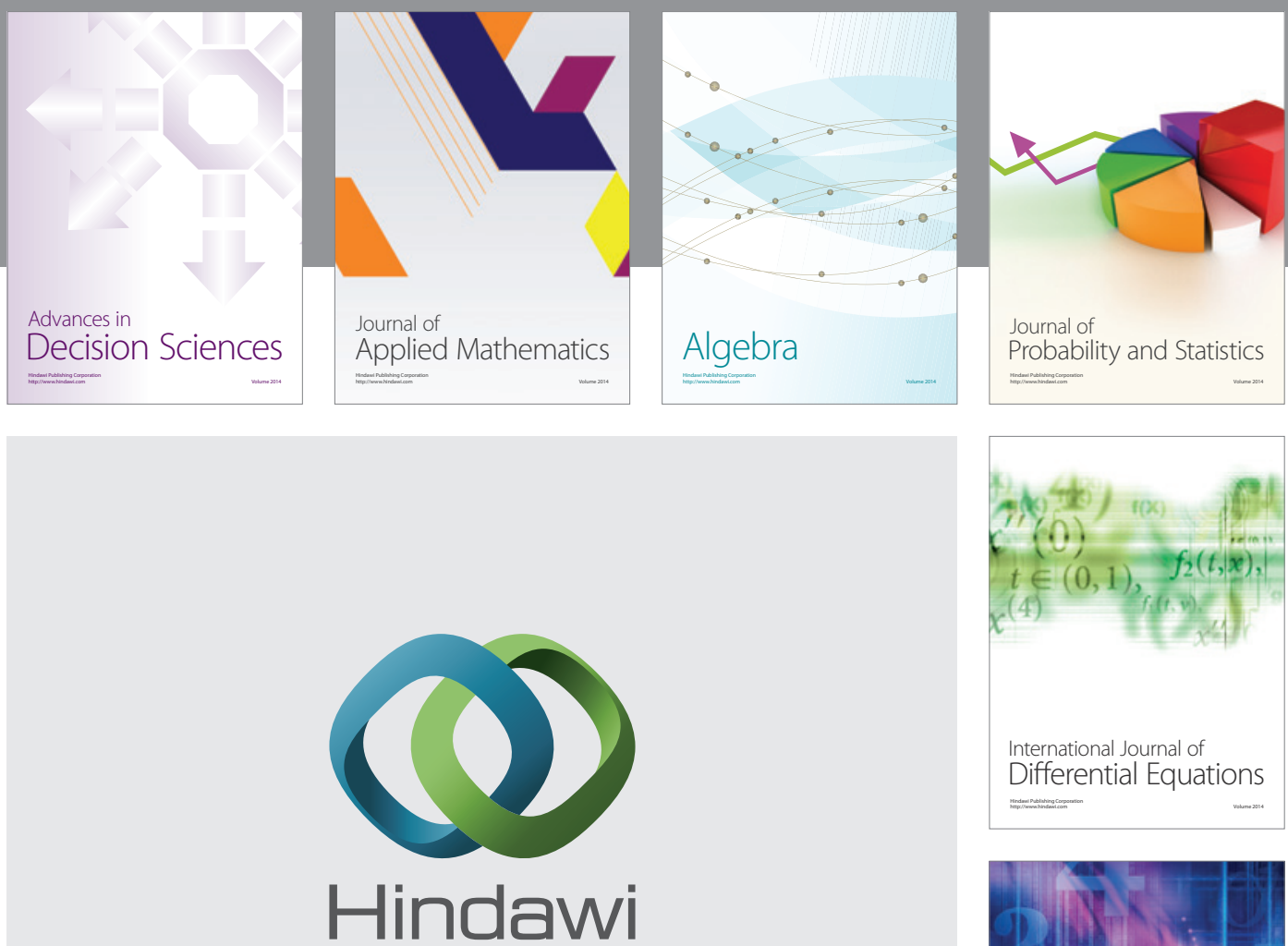

Submit your manuscripts at http://www.hindawi.com
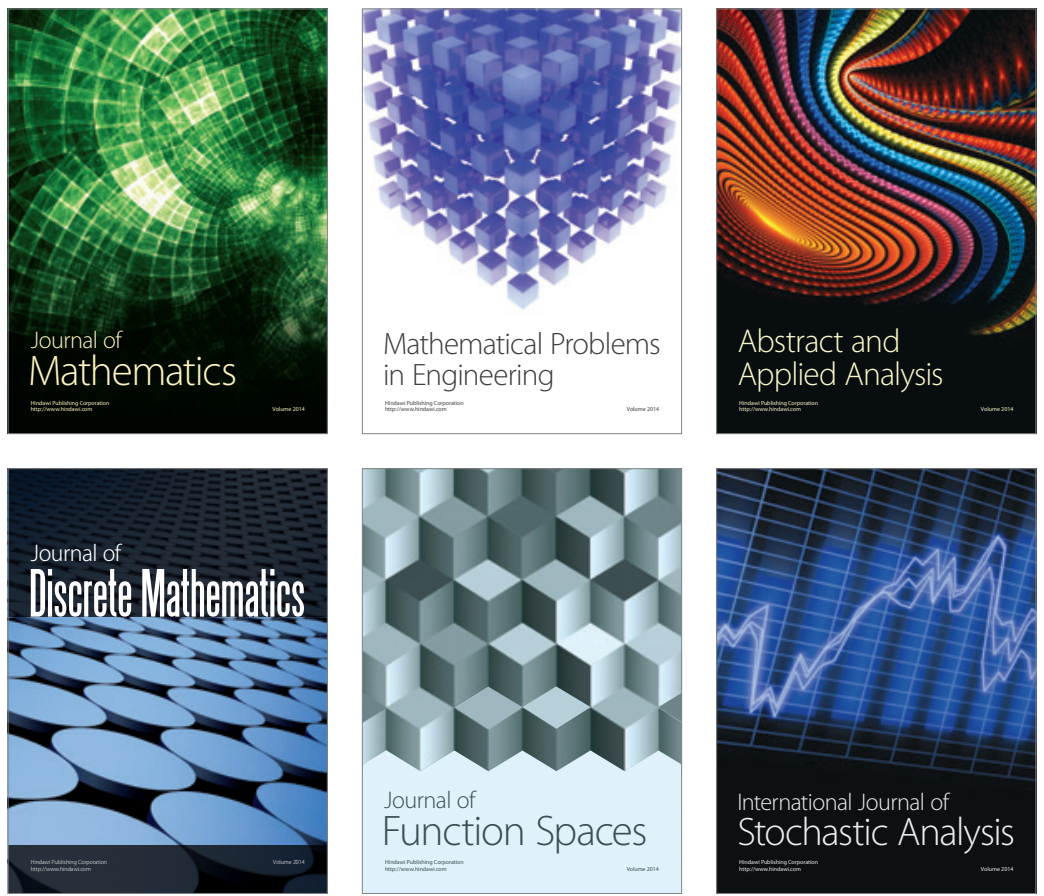

Journal of

Function Spaces

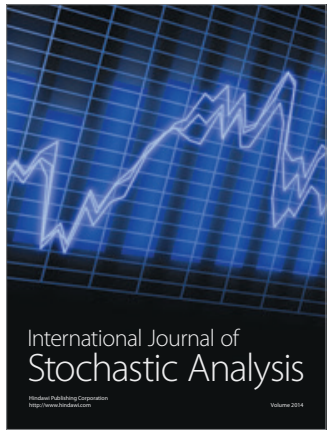

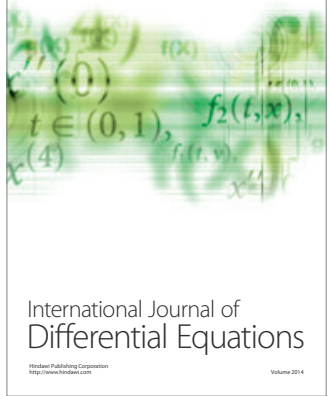
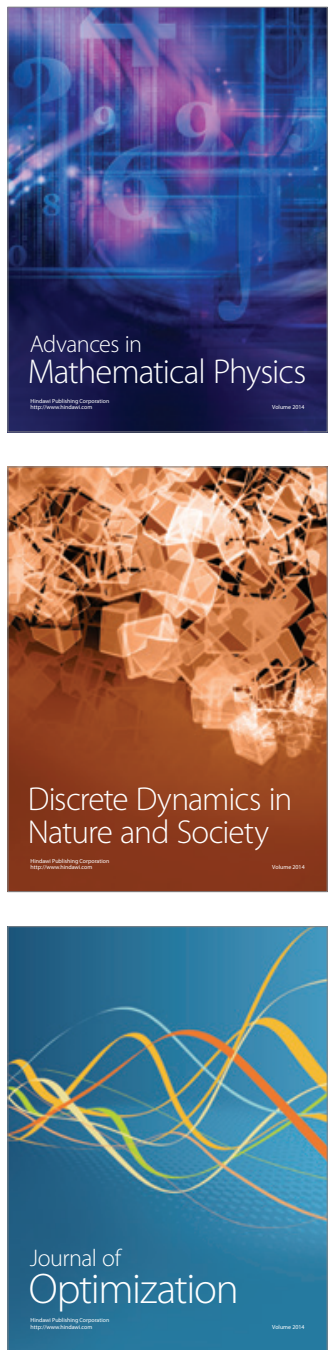